\title{
The resilience metric investigation of vibration reduction for rectangular thin plate system excited by multi-vibration sources
}

\author{
Guoxing Zhu ${ }^{1, a}$ Changli Liu ${ }^{1, b}$ and Fei Song ${ }^{1, c}$, \\ ${ }^{1}$ School of Mechanical and Power Engineering, East China University of Science and Technology, \\ Shanghai 200237, China \\ azgx920531@163.com, bclliu@ecust.edu.cn, cflysong110@163.com
}

Keywords: resilience metric, state recovery, rectangular thin plate system, vibration reduction

\begin{abstract}
Resilience often refers to a property of social and ecological systems, recently, resilience is applied to the engineered system, referring to their capability to recover the state after original state degrade from external disturbance. In this paper, a resilience metric is proposed corresponding to vibration reduction of rectangular thin plate system excited by multi-vibration sources. A main purpose of this paper is to provide a methodological guidance for measuring resilience of engineering system.
\end{abstract}

\section{Introduction}

The first use of resilience concept in the academic work could be classified into material science [1]. In the 19th century, Tredgold [2] used the resilience concept to represent the timber's property of sustaining sudden and severe loads without breaking. Mallet [3] [4] also used the resilience concept to describe the performance of materials under severe conditions and discuss the suitability of materials in the construction of vessels and buildings The second application of resilience concept was in ecology field, Holling [5] introduced it into the ecology and redefined it: resilience is a characteristic of ecology system which enables system to absorb the external environment change and still maintain its function. After that, the application of resilience from a specific discipline to a wider range of domains, including: organizational domain, social domain, economic domain, engineering domain.

Compared to the three other domains, the resilience concept used in engineering domain is still a new research direction. In the context of engineering, the resilience can be described as: a property of a system on how can the system still function to a desired level by its own self-repairing when the system suffers from a partial damage [6]. As can be seen by the above description, one feature of resilient engineering system is self-repairing, instead of relying on extra resources, so, according to this feature, we can propose a resilient analysis method for the state recovery of engineering system after its normal state is damaged.

In the different aspects of resilient analysis method, many scholars focus on the problem of resilience metric, that is how to measure a system's state recovery ability. In this paper, we propose a resilience metric method which is related with recovery effect of system state, the resilience metric of rectangular thin plate system excited by multi-vibration sources is studied with this method. The state of studied engineering system refers to the kinetic energy magnitude of rectangular thin plate, system state will degrade with kinetic energy increasing, we choose to recover the state of rectangular thin plate system by configuring the different locations of multi-vibration sources.

\section{Model Building}

In this paper, the rectangular thin plate system is studied, and the plate kinetic energy is regarded as system state. In order to understand the rectangular thin plate system for resilience analysis, it is necessary to model the studied system, so that, the modeling can provide the theoretical basis for the next resilience metric analysis. The following is the detailed modeling process. 
Assuming the rectangular thin plate is subject to $\mathrm{S}+1$ excitation forces, for the convenience of research, one of the forces is regarded as original force $f_{p}=F_{0} e^{j \omega t}$ while others are regarded as control forces $f_{c k}=F_{0} e^{j\left(\omega t+\varphi_{c k}\right)},(k=1,2, \cdots S)$, where $\varphi_{c k}$ is phase differences between original force and control forces, $\left(x_{p}, y_{p}\right)$ and $\left(x_{c k}, y_{c k}\right)$ are the coordinates of original and control forces.

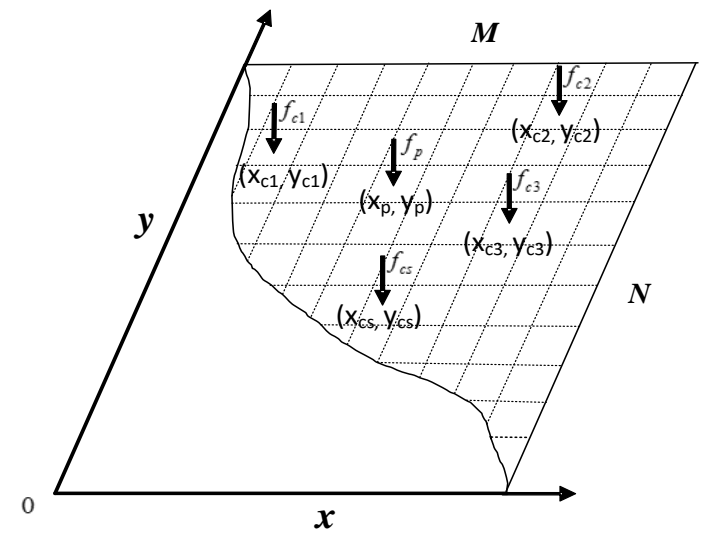

Fig.1 Theoretical Analysis Model

As shown in Fig.1, the mathematical model built in for the simulation considers the plate divided into a grid of rectangular elements whose dimensions have been taken to be $l_{x e}=\frac{a}{M}, l_{y e}=\frac{b}{N}$, where $\mathrm{M}$ and $\mathrm{N}$ are the plate modal orders used in the calculus. The central coordinate of each elements is $\left(x_{e}, y_{e}\right)$, where $x_{e}=\frac{(2 i-1) a}{2 M}, y_{e}=\frac{(2 j-1) b}{2 N}(i=1,2,3, \cdots, M ; j=1,2,3, \cdots, N)$. The phasors of the control force $F_{c}$ is:

$$
F_{c}=\left(\begin{array}{c}
f_{c 1} \\
f_{c 2} \\
\vdots \\
f_{c S}
\end{array}\right)
$$

The vibration of the plate at the centers of the elements can be expressed in matrix form using mobility functions:

$$
v_{e}=Y_{p, e} f_{p}+Y_{c, e} F_{c}
$$

Where the components of the velocity/force mobility matrices, $Y_{p, e}, Y_{c, e}$, between the position of forces and elements, are given by:

$$
Y_{p(c), e}=\frac{4 j \omega}{\rho h a b} \sum_{m=1}^{\infty} \sum_{n=1}^{\infty} \frac{\sin \left(\frac{m \pi x_{p(c),}}{a}\right) \sin \left(\frac{n \pi y_{p(c)},}{b}\right) \sin \left(\frac{m \pi x_{e}}{a}\right) \sin \left(\frac{n \pi y_{e}}{b}\right)}{\omega_{m n}^{2}(1+j \delta)-\omega^{2}}
$$

Where $\rho, h, a, b$ is density, thickness, length and width of the plate. $j=\sqrt{-1} . \delta$ is the hysteresis loss factor. $m, n$ is the modal indices. $\omega_{m n}=\pi^{2}\left(\frac{m^{2}}{a^{2}}+\frac{n^{2}}{b^{2}}\right) \sqrt{\frac{D}{\bar{m}}}$ is the natural frequency of the $(m, n) t h$ bending mode where $\bar{m}=\rho h$ is surface density of elements, $D=\frac{E h^{3}}{12\left(1-\mu^{2}\right)}$ is stiffness of the plate where $\mathrm{E}$ is Modulus of Elasticity and $\mu$ is Poisson ratio.

This expression can be approximated to the summation of the kinetic energies of each element into which the plate has been subdivided so that,

$$
\begin{gathered}
E_{e}(\omega)=\frac{M_{e}}{4} v_{e}^{H} v_{e} \\
E(\omega)=\sum_{i=1}^{M} \sum_{j=1}^{N} E_{e}(\omega)
\end{gathered}
$$

Where $M_{e}=\rho h l_{x e} l_{y e}$ is the mass of the elements and $H$ denotes the Hermitian transpose. 


\section{Resilience metric}

According to the analysis of previous section, the kinetic energy of rectangular thin plate is the function of vibration sources coordinates, that is to say we can configure the different locations of vibration sources to recover system state if its vibration seriously. In order to facilitate the following resilience metric research, we make two assumptions: 1 . The number of vibration sources is just two, that is one original force and one control forces. 2 . When the kinetic energy of rectangular thin plate is larger than that of one vibration source acting on the plate, we consider the state of rectangular thin plate system is degraded.

Regarding the resilience metric of engineering system, Wang $\mathrm{J}$ [7] proposed three important axioms:

Axiom 1: The resilience of a system can only be measured for a particular imbalanced situation.

Axiom 2: A system which can rebalance the supply and demand relation from a larger imbalanced situation is more resilient.

Axiom 3: A system which can rebalance the supply and demand relation from the imbalanced situation with less time is more resilient.

As a result, the resilience metric of rectangular thin plate system can be determined by state recovery effect, in other words, the change of kinetic energy after configuring a reasonable vibration sources location reflects system state effect. At last, the resilience metric of rectangular thin plate system can be calculated with Eq. (5).

$$
\mathrm{I}\left(E_{k \mathrm{~s}}, E_{k 0}\right)=\frac{E_{k s}-E_{k 0}}{E_{k 0}}
$$

Where $\mathrm{I}$ is resilience metric, $E_{k s}, E_{k 0}$ are kinetic energy after recovery and before recovery respectively. $E_{k s}, E_{k 0}$ can be calculated by Eq. (4).



Fig.2 The configuration of plate before recovery

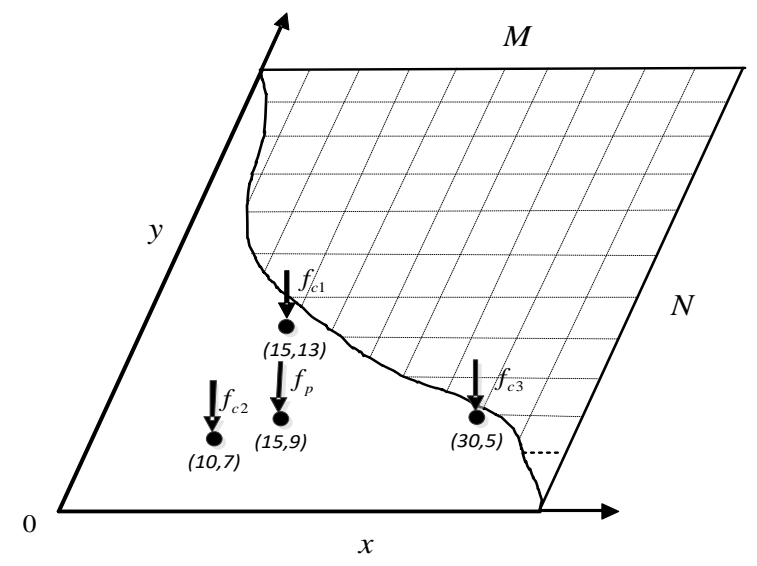

Fig.3 The configuration of plate after recovery

Take an example to illustrate the calculated process of proposed resilience metric. Assuming in a certain condition, the coordinate of original force is $(15,9)$, the coordinate of control force is $(20,13)$ acting on the rectangular thin plate, as shown in Fig.2. the standard kinetic energy with one original force $(15,9)$ acting on the plate is $E_{k 0}=-98 D B$, the initial kinetic energy with two forces $(15,9),(20,13)$ acting on the plate is $E_{k 1}=-72 D B$, since, $E_{k 1}>E_{k 0}$, the state of rectangular thin plate is degraded. In this case, on the basis of the analysis process of modeling the rectangular thin plate system, we can know that reconfiguration the locations of vibration sources can change the kinetic energy for state recovery, hence, if change the coordinate of control force to three configurations, which are $(15,13)$, $(10,7),(30,5)$ respectively, the different kinetic energy of rectangular thin plate system can be obtained. Fig.3 presents the state recovery strategies. The changed kinetic energy after state recovery are: $E_{k 2}=-108 D B, E_{k 2}=-200 D B, E_{k 3}=-135 D B$. Then, the resilience metric under different recovery strategies can be calculated by Eq. (5) are: 


$$
\begin{aligned}
& I_{1}=\frac{E_{k 2}-E_{k 0}}{E_{k 0}}=\frac{-108-(-98)}{-98}=0.1 \\
& I_{2}=\frac{E_{k 3}-E_{k 0}}{E_{k 0}}=\frac{-200-(-98)}{-98}=1.0 \\
& I_{3}=\frac{E_{k 4}-E_{k 0}}{E_{k 0}}=\frac{-135-(-98)}{-98}=0.4
\end{aligned}
$$

The larger the value, the better the effect, the better the state recovery. Tab.1 lists the different resilience metrics with different recovery effect.

Tab.1 The different resilience metrics with three configurations of vibration sources

\begin{tabular}{|c|c|c|c|c|}
\hline $\begin{array}{c}\text { The coordinate } \\
\text { of original force } \\
\left(x_{p}, y_{p}\right)\end{array}$ & $\begin{array}{c}\text { Standard kinetic } \\
\text { energy } \\
\left(\mathrm{E}_{k 0}\right)\end{array}$ & $\begin{array}{c}\text { The coordinates of control } \\
\text { force with different } \\
\text { configurations } \\
\left(x_{c}, y_{c}\right)\end{array}$ & $\begin{array}{c}\text { The changed kinetic } \\
\text { energy after state } \\
\text { recovery } \\
\left(\mathrm{E}_{k \mathrm{~s}}\right)\end{array}$ & $\begin{array}{c}\text { Resilience } \\
\text { metric } \\
(\mathrm{I})\end{array}$ \\
\hline$(15,9)$ & $-98 \mathrm{DB}$ & $(15,13)$ & $-108 \mathrm{DB}$ & 0.1 \\
\hline$(15,9)$ & $-98 \mathrm{DB}$ & $(10,7)$ & $-200 \mathrm{DB}$ & 1 \\
\hline$(15,9)$ & $-98 \mathrm{DB}$ & $(30,5)$ & $-135 \mathrm{DB}$ & 0.4 \\
\hline
\end{tabular}

\section{Conclusions}

In this paper, theoretical analysis and numerical calculation are presented to research the resilience metric of vibration reduction for rectangular thin plate system excited by multi-vibration sources. From the above analysis process of resilience metric, we can draw a conclusion: every vibration reduction strategy represents the different system recovery effects, and the resilience metric calculated by Eq. (5) can reflects the different state recovery effects of rectangular thin plate system.

\section{Acknowledgments}

The authors are grateful to the China Natural Science Funds (NSFC, Grant No. 51175179), and the Fundamental Research Funds for the Central Universities of China

\section{References}

[1] McAslan A. The concept of resilience: Understanding its origins, meaning and utility[J]. Adelaide: Torrens Resilience Institute, 2010.

[2] Tredgold T. On the transverse strength and resilience of timber[M]. Taylor \& Francis, 1818.

[3] Mallet R. On the Physical Conditions Involved in the Construction of Artillery: With an Investigation of the Relative and Absolute Values of the Materials Principally Employed, and of Some Hitherto Unexplained Causes of the Destruction of Cannon in Service[M]. Longmans, Brown, Green, Longmans, and Roberts, 1856.

[4] Mallet R. The First Principles of Observational Seismology: As Developed in the Report to the Royal Society of London of the Expedition Made by Command of the Society Into the Interior of the Kingdom of Naples, to Investigate the Circumstances of the Great Earthquake of December 1857[M]. Chapman and Hall, 1862.

[5] Holling C S. Resilience and stability of ecological systems[J]. Annual review of ecology and systematics, 1973: 1-23.

[6] Zhang W J, Lin Y. On the principle of design of resilient systems-application to enterprise information systems[J]. Enterprise Information Systems, 2010, 4(2): 99-110.

[7] Wang J. Towards a resilient networked service system[J]. 2013. 